\title{
Surface improvement of organic photoresists using a near-field-dependent etching method
}

\author{
Felix J. Brandenburg ${ }^{1}$, Tomohiro Okamoto ${ }^{1}$, Hiroshi Saito ${ }^{1}$, Benjamin Leuschel $^{2}$, \\ Olivier Soppera ${ }^{2}$ and Takashi Yatsui ${ }^{* 1}$
}

\author{
Full Research Paper \\ Address: \\ ${ }^{1}$ School of Engineering, University of Tokyo, Bunkyo-ku, Tokyo, \\ 113-8656, Japan and ${ }^{2}$ Institut de Sciences des Materiaux de \\ Mulhouse (IS2M), CNRS UMR 7361, 15 rue Jean Starcky, BP 2488, \\ Mulhouse Cedex 68057, France \\ Email: \\ Takashi Yatsui* - yatsui@ee.t.u-tokyo.ac.jp \\ * Corresponding author \\ Keywords: \\ near-field etching; organic photoresists; surface improvement; \\ wavelength dependence
}

Beilstein J. Nanotechnol. 2017, 8, 784-788.

doi:10.3762/bjnano.8.81

Received: 12 January 2017

Accepted: 23 March 2017

Published: 05 April 2017

Associate Editor: P. Leiderer

(C) 2017 Brandenburg et al.; licensee Beilstein-Institut.

License and terms: see end of document.

\begin{abstract}
Surface flattening techniques are extremely important for the development of future electrical and/or optical devices because carrier-scattering losses due to surface roughness severely limit the performance of nanoscale devices. To address the problem, we have developed a near-field etching technique that provides selective etching of surface protrusions, resulting in an atomically flat surface. To achieve finer control, we examine the importance of the wavelength of the near-field etching laser. Using light sources at wavelengths of 325 and $405 \mathrm{~nm}$, which are beyond the absorption edge of the photoresist (310 $\mathrm{nm})$, we compare the resulting cross-sectional etching volumes. The volumes were larger when $325 \mathrm{~nm}$ light was employed, i.e., closer to the absorption edge. Although $405 \mathrm{~nm}$ light did not cause structural change in the photoresist, a higher reduction of the surface roughness was observed as compared to the $325 \mathrm{~nm}$ light. These results indicate that even wavelengths above $325 \mathrm{~nm}$ can cause surface roughness improvements without notably changing the structure of the photoresist.
\end{abstract}

\section{Introduction}

As the structures fabricated in the field of semiconductors has reached below $10 \mathrm{~nm}$ [1], and the pursuit of ever smaller node sizes continues, the impact of the surface roughness (SR) becomes increasingly critical [2]. In attempts to downscale the minimum feature size year-by-year in order to sustain Moore's Law $[3,4]$, new processing techniques [5] as well as materials [6] are constantly being explored. One important criterion for new materials and techniques is their SR value, and the reduction of the SR is of great interest for high-end lithography.

Conventional chemical mechanical polishing (CMP) methods [7] are generally limited by the roughness of its polishing pad, which is on the order of $10 \mu \mathrm{m}$, and by the diameter of the particles in the chemical slurry, which can be around $100 \mathrm{~nm}$. 
Furthermore, due to issues with cost and material availability, there have been efforts to reduce the usage of the rare-earth material $\mathrm{CeO}_{2}$ used in the chemical slurry of CMP [8]. So in order to achieve SR reduction without the use of CMP methods, a novel approach, called near-field etching, has been introduced. This fine-tuning technique has previously proven to be effective in producing atomically flat surfaces in various materials, including GaN [9], glass [10] and even diamond [11] and has shown to be effective on both flat and patterned surfaces.

According to theory, the etching effect originates from radical gas-phase components. More precisely, ambient $\mathrm{O}_{2}$ molecules can be dissociated at $5.12 \mathrm{eV}$ [12], and these radical $\mathrm{O}$ atoms react with the surface of a specimen. However, it is important to keep in mind that the laser energy used is below $5.12 \mathrm{eV}$ $(242 \mathrm{~nm})$ and therefore does not directly cause the dissociation. The etching laser wavelength $(325 \mathrm{~nm})$ is carefully chosen to be below the direct $\mathrm{O}_{2}$ dissociation energy. Previous theories suggest localized optical near-fields can cause two-step excitation via vibrational levels in molecules [13]. In theory, the localized optical near-field has a nonuniform field distribution and thus can activate the dipole-forbidden intermediate vibrational states of molecules. In other words, although the sample is excited using a laser of energy less than $5.12 \mathrm{eV}$, owing to the vibrational states, the energy eventually increases to $5.12 \mathrm{eV}$ because of the excitation of localized photons. Furthermore, these localized photons are believed to be primarily present in the protrusions on the surface. Thus, $\mathrm{O}_{2}$ dissociation, and hence etching, occurs primarily at locations with a high protrusion density. Previous research suggests that near-field-based energy upconversion can also occur through multiphoton absorption instead of single-photon absorption but the former has a much lower probability $[14,15]$.

The photoresist in this study is a conventional, organic, chemically amplified resist (CAR) (EPIC 2096 ArF Photoresist), which is sensitive to $\operatorname{ArF}$ excimer laser excitation $(\lambda=193 \mathrm{~nm})$. Organic photoresists are easily obtained and play an important role in high-end integrated circuit (IC) chip production. Since the quality of IC semiconductors is directly dependent on their $\mathrm{SR}$, an improvement in the structure of the photoresist during the lithography process could significantly improve the quality of the semiconductor end-product. Furthermore, since near-field etching has only been tested on nonorganic, flat materials and nonpatterned organic materials, it is of great interest to observe the near-field etching effect on organic photoresists.

\section{Experimental}

For the purpose of this study, a positive tone organic photoresist was prepared using interference lithography. Specifically, the chemically amplified resist (EPIC 2096 ArF Photoresist) with an absorption edge of $310 \mathrm{~nm}$ was used. To observe the wavelength dependence, the continuous-wave $(\mathrm{CW})$ laser sources used in these experiments were: (1) $\mathrm{He}-\mathrm{Cd}$ laser (325 nm; $3.81 \mathrm{eV}$; excitation power density: $0.8 \mathrm{~W} / \mathrm{cm}^{2}$ ) and (2) $\mathrm{GaN}$ laser (405 nm; $3.06 \mathrm{eV}$; excitation power: $39 \mathrm{~mW}$ ). These lasers were carefully selected to be below the dissociation energy of $\mathrm{O}_{2}(5.12 \mathrm{eV})$ in order to avoid conventional photochemical etching through direct $\mathrm{O}_{2}$ dissociation. The light intensity for all sources except $325 \mathrm{~nm}$ was $10^{-4}$ times greater than the $325 \mathrm{~nm}$ source. Therefore, the emission line (except for $325 \mathrm{~nm}$ ) was negligibly low in intensity and could not have dissociated the $\mathrm{O}_{2}$ molecules. The photoresist was illuminated by the respective lasers for a specific time $(0-120 \mathrm{~min})$. For the vacuum experiment, before illumination, the sample was fixed into a glass box connected to a vacuum pump. Through a purification process, the $\mathrm{O}_{2}$ density inside the glass box was reduced to approximately tens of ppb by first removing the air with the vacuum pump and then refilling the box with $\mathrm{N}_{2}$ (containing $\mathrm{O}_{2}$ at $5 \mathrm{ppb}$ ) until the starting pressure was restored. This process was repeated 10 times, resulting in a low-oxygen (room pressure) environment. The sample surfaces were evaluated using atomic force microscopy (AFM) (Hitachi-Hitech-Science Corp.). The scanned areas were around 3-4 $\mu \mathrm{m}$ in area with a resolution of $256 \times 256$ pixels. In the AFM software, the "sample intelligent scan" mode was used during the measurement and images were improved using the tilt compensation features. Surface roughness values were automatically obtained by the AFM software, which averaged values of the absolute surface height deviations from a best-fitting plane.

\section{Results and Discussion}

In order to test the effectiveness of near-field etching, the photoresist substrate was scanned by AFM before and after laser illumination (Figure 1). Figure 1a and Figure 1b show the photoresist before and after near-field etching with a $325 \mathrm{~nm}$ laser, respectively. Figure 1c and Figure 1d show the photoresist before and after near-field etching with a $405 \mathrm{~nm}$ laser, respectively. The samples were prepared under ambient room conditions, since $\mathrm{O}_{2}$ molecules are believed to be integral to this etching phenomenon. Figure 1a and Figure $1 \mathrm{~b}$ clearly show that illuminating the photoresist with $325 \mathrm{~nm}$ laser light changes the structural shape of the photoresist. The images indicate that the photoresist becomes thinner after being illuminated with $325 \mathrm{~nm}$ light. The photoresist exposed to near-field etching with $405 \mathrm{~nm}$ light in Figure 1c and Figure 1d does not change its structural shape noticeably; however, the surface texture of the photoresist appears to become smoother after near-field etching when looking at the detailed images in Figure 1e and Figure 1f.

For a more detailed view, Figure 2a illustrates the crosssectional profile of the photoresist as a function of $325 \mathrm{~nm}$ light 

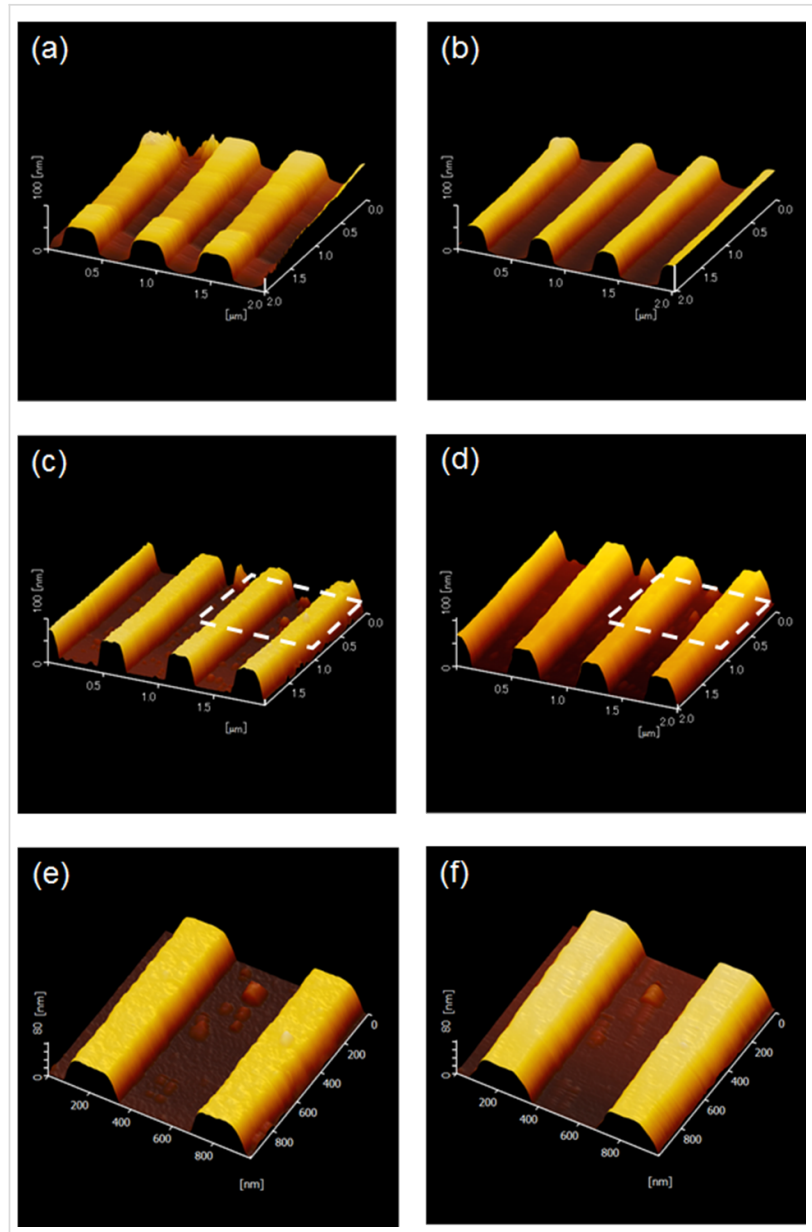

Figure 1: AFM images of the photoresist after $120 \mathrm{~min}$ of near-field etching with a He-Cd laser ( $325 \mathrm{~nm}, 3.81 \mathrm{eV})$ ((a) before and (b) after) and a GaN laser (405 nm; $3.06 \mathrm{eV}$ ) ((c) before and (d) after). (e) and (f) provide details of (c) and (d) respectively at the same positions (white dashed line).

illumination time. Over this time, the mid-section of the photoresist was reduced from approximately $400 \mathrm{~nm}$ to $250 \mathrm{~nm}$ in width. In Figure $2 b$ we can see no noticeable change in the cross-sectional profile of the photoresist after near-field etching with $405 \mathrm{~nm}$ light. While both laser wavelengths $(325 \mathrm{~nm}$ and $405 \mathrm{~nm}$ ) were longer than the $\mathrm{O}_{2}$ dissociation wavelength $(242 \mathrm{~nm}$ ) (in order to avoid adiabatic etching), the $325 \mathrm{~nm}$ laser excitation appeared to have induced an etching reaction, but the $405 \mathrm{~nm}$ laser did not. However the results do not exclude an $\mathrm{O}_{2}$ dissociation reaction through the $405 \mathrm{~nm}$ laser entirely, if we consider the change of surface smoothness between Figure 1c and Figure 1d. Because the energy of the $405 \mathrm{~nm}$ laser $(3.06 \mathrm{eV})$ was significantly lower than the dissociation energy of $\mathrm{O}_{2}$, it can be assumed that the $405 \mathrm{~nm}$-laser-induced dissociation of $\mathrm{O}_{2}$ molecules is probably attributable to the phenomenon of multiphoton absorption, which occurs far less frequently. In general, it is important to note that the phenomenon of nearfield-caused multiphoton absorption is unrelated to the conventional far-field-caused multiphoton absorption. A photon from the $325 \mathrm{~nm}$ laser, on the other hand, triggers the dissociation of $\mathrm{O}_{2}$ far more readily because its energy is much higher than that of a photon from the $405 \mathrm{~nm}$ laser.

In order to evaluate the degree of surface smoothening by each laser, we evaluated the surface roughness before and after etching through a program embedded in the AFM in Figure 3a. Both lasers were able to smooth the photoresist by reducing its surface roughness over an interval of $2 \mathrm{~h}$. Owing to the nature of the samples, as well as that of the AFM measurement process, fluctuations in the initial SR values were unavoidable. This is the reason for the observed differences in the starting SR values. Although the smoothing rates of the two lasers should not be compared with each other directly, the rate of the $325 \mathrm{~nm}$ laser was slightly lower than that of the $405 \mathrm{~nm}$ laser. In the case of the $405 \mathrm{~nm}$ laser, the difference might be caused by a stronger presence of optical near-fields, owing to the larger initial SR value of the corresponding sample. However, another possible reason could be that the $325 \mathrm{~nm}$ laser, through its high etching rate, is removing the surface layers rather than
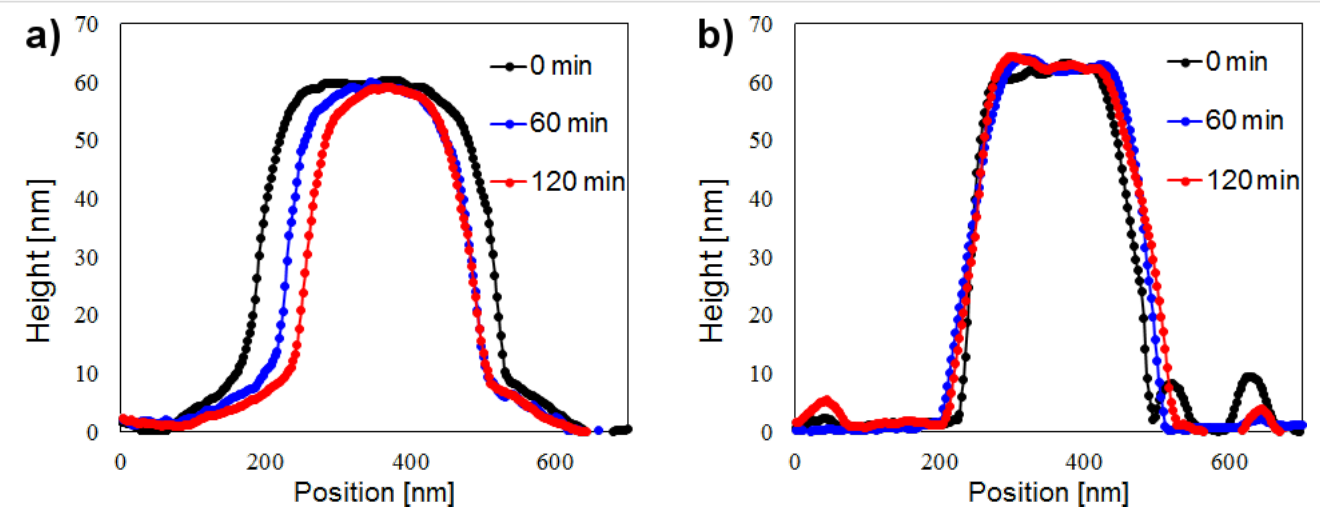

Figure 2: (a) Photoresist profile before (black) and after $60 \mathrm{~min}$ (blue) and $120 \mathrm{~min}$ (red) of $325 \mathrm{~nm}$ laser illumination under room conditions. (b) Same as (a) but with $405 \mathrm{~nm}$ laser illumination. 


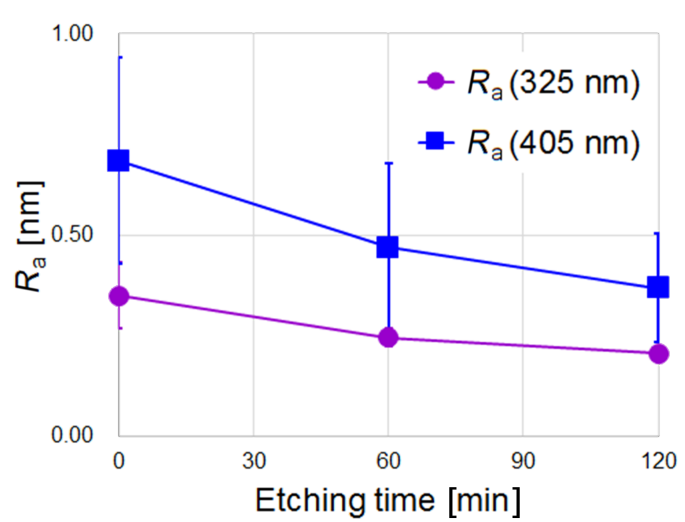

(a)

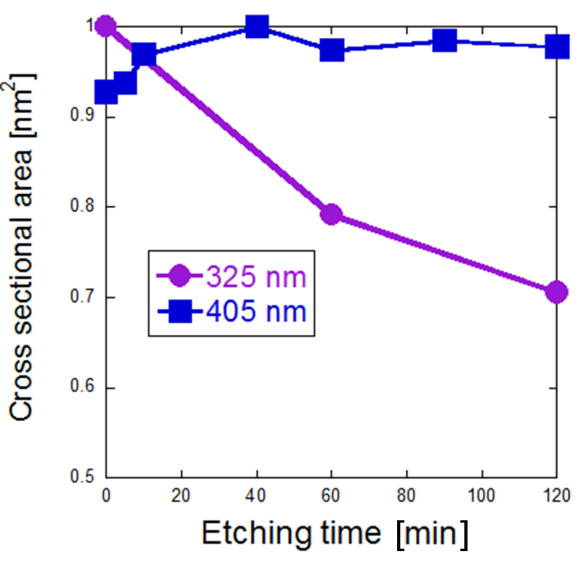

(b)

Figure 3: (a) Evaluation of surface roughness reduction of $325 \mathrm{~nm}$ (purple) and $405 \mathrm{~nm}$ (blue) over a $2 \mathrm{~h}$ interval. (b) Showing the cross-sectional etching volume. A comparison of the etching volumes of $325 \mathrm{~nm}$ (purple) and $405 \mathrm{~nm}$ (blue) over a $2 \mathrm{~h}$ interval.

smoothing them (rough versus fine etching), as can be seen in Figure $3 \mathrm{~b}$. The $405 \mathrm{~nm}$ laser, on the other hand, does not seem to remove any layers of the photoresist. It is mostly smoothing the same outermost layer, hence resulting in a higher surface roughness reduction rate. Again, here we must differentiate between the surface roughness reduction or smoothing rate (Figure 3a) and the gross etching rate (Figure 3b), and how each laser wavelength appears to have comparatively different rates for them.

Furthermore, the results in Figure 2 and Figure 3 indicate that the etching effect of near-field etching is not linear, but actually diminishes over time. Possible explanations for this are that the near-field etching reduces the surface roughness, meaning that after a specific duration there could be less surface protrusions. This seems to be a general condition for near-field etching and is a plausible one since a relative decrease in the number of protrusions during the etching process means a decrease in the generation of the local near-fields as well. The saturation effect has been observed in a wide range of samples and seems to be material independent.

In order to prove that the structural changes are not caused directly by the inherent UV-light sensitivity of the EPIC 2096 ArF photoresist, an absorption spectrum measurement was performed under ambient room conditions (Figure 4a). The spectrum shows that there is no absorption of 325 or $405 \mathrm{~nm}$ light. Hence, the inherent UV-light sensitivity of the photoresist can be disregarded as a factor in this paper. Additionally, the same etching experiment (as in Figure 2a) was repeated under a lowoxygen environment, with the result depicted in Figure $4 \mathrm{~b}$. Under these conditions, it was not possible to replicate the

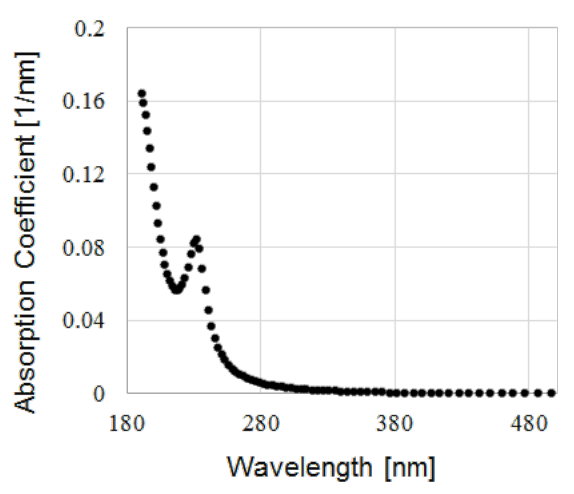

(a)

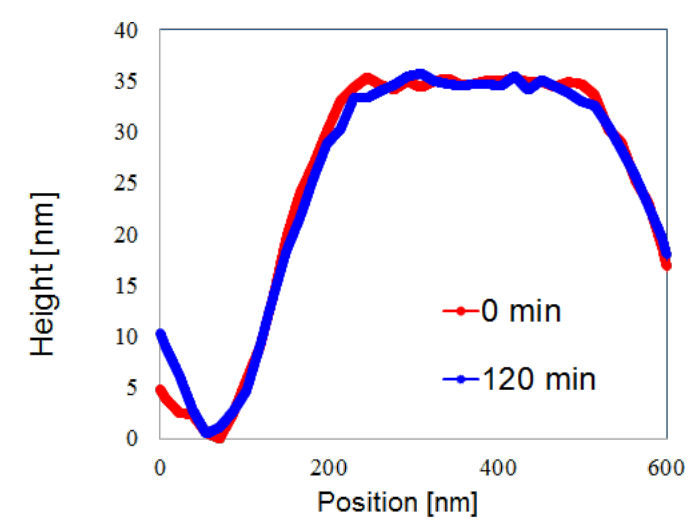

(b)

Figure 4: Absorption spectrum and cross-sectional profile. (a) Photoresist absorption curve, showing that $325 \mathrm{~nm}$ and $405 \mathrm{~nm}$ are not directly being absorbed by the photoresist. (b) Near-field etching for $120 \mathrm{~min}$ with $325 \mathrm{~nm}$ He-Cd laser, repeated under a low-oxygen environment. 
width loss in Figure 2a. This gives a further reason to assume that radical $\mathrm{O}$ atoms are the general cause of the etching phenomena, as well as ruling out the possibility of inherit absorption effects of the photoresist in the case of the $325 \mathrm{~nm}$ laser, as shown in Figure 4a. To sum up, the data in Figure $4 \mathrm{a}$ and Figure $4 \mathrm{~b}$ indicates that the etching phenomena is somewhat dependent on ambient $\mathrm{O}_{2}$ molecules and that the structural change is not due to the natural UV sensitivity of the photoresist.

\section{Conclusion}

It was possible to change the shape of the photoresist by nearfield etching, although the etching ratio is shown to diminish over the etching time. Here, we show that $325 \mathrm{~nm}$ illumination changes the cross-sectional width of the photoresist to a greater extent than $405 \mathrm{~nm}$ illumination. Conversely, $405 \mathrm{~nm}$ light reduced surface roughness to a greater extent than $325 \mathrm{~nm}$ light. Overall, this paper proves that near-field etching, through its noncontact nature, could be critical for the development of future nanoscale devices. Furthermore, the results indicate that there is a wavelength dependence of near-field etching, and it would be interesting to further investigate this dependence by examining a wider range of etching wavelengths.

\section{Author Contributions}

T.Y. planned the project. F.B. and T.O. performed near-field etching experiments. B.L., H.S. and O.S. prepared organic photoresist samples. All authors discussed the results. F.B. and T.Y. wrote the manuscript. All authors reviewed the manuscript.

\section{Acknowledgements}

The authors wish to express special thanks to Prof. Katsuyuki Nobusada (Institute for Molecular Science) for active support and discussion. This work was partially supported by MEXT Grant-in-Aid for Program for Leading Graduate Schools (Materials Education Program for the Future Leaders in Research, Industry, and Technology: MERIT), a MEXT Grantin-Aid for Scientific Research (B) (No. 26286022), a MEXT Grant-in-Aid for Grant-in-Aid for Scientific Research on Innovative Areas (No. 15H00866), a MEXT Nanotechnology Platform (No.12024046), the JSPS Core-to-Core Program (A. Advanced Research Networks), the JSPS Japan (JSPS)-Korea (NRF) Bilateral Program, the Yazaki Memorial Foundation, and the Research Foundation for Opto-Science and Technology.
2. Park, B. C.; Cho, Y. C.; Kim, I.; Yeo, J. Proc. SPIE 2013, 8681, 86812K. doi:10.1117/12.2011677

3. Tallents, G.; Wagenaars, E.; Pert, G. Nat. Photonics 2010, 4, 809-811. doi:10.1038/nphoton.2010.277

4. Wagner, C.; Harned, N. Nat. Photonics 2010, 4, 24-26. doi:10.1038/nphoton.2009.251

5. Ok, J. G.; Shin, Y. J.; Park, H. J.; Guo, L. J. Appl. Phys. A 2015, 121, 343-356. doi:10.1007/s00339-015-9229-6

6. Oleksak, R. P.; Ruther, R. E.; Luo, F.; Fairley, K. C.; Decker, S. R.; Stickle, W. F.; Johnson, D. W.; Garfunkel, E. L.; Herman, G. S.; Keszler, D. A. ACS Appl. Mater. Interfaces 2014, 6, 2917-2921. doi:10.1021/am405463u

7. Jianfeng, L.; Dornfeld, D. A. IEEE Trans. Semicond. Manuf. 2001, 14, 112-133. doi:10.1109/66.920723

8. Kato, Y.; Fujinaga, K.; Nakamura, K.; Takaya, Y.; Kitamura, K.; Ohta, J.; Toda, R.; Nakashima, T.; Iwamori, H. Nat. Geosci. 2011, 4, 535-539. doi:10.1038/ngeo1185

9. Yatsui, T.; Nomura, W.; Stehlin, F.; Soppera, O.; Naruse, M.; Ohtsu, M. Beilstein J. Nanotechnol. 2013, 4, 875-885. doi:10.3762/bjnano.4.99

10. Nomura, W.; Yatsui, T.; Kawazoe, T.; Tate, N.; Ohtsu, M. Appl. Phys. A 2015, 121, 1403-1407. doi:10.1007/s00339-015-9466-8

11. Yatsui, T.; Nomura, W.; Naruse, M.; Ohtsu, M. J. Phys. D: Appl. Phys. 2012, 45, No. 475302. doi:10.1088/0022-3727/45/47/475302

12. Keilin, D.; Hartree, E. F. Nature 1950, 165, 543-544. doi:10.1038/165543a0

13. Yamaguchi, M.; Nobusada, K. Phys. Rev. A 2016, 93, No. 023416. doi:10.1103/PhysRevA.93.023416

14. Yatsui, T.; Imoto, T.; Mochizuki, T.; Kitamura, K.; Kawazoe, T. Sci. Rep. 2014, 4, 4561. doi:10.1038/srep04561

15. Yamaguchi, M.; Nobusada, K.; Kawazoe, T.; Yatsui, T. Appl. Phys. Lett. 2015, 106, 191103. doi:10.1063/1.4921005

\section{License and Terms}

This is an Open Access article under the terms of the Creative Commons Attribution License (http://creativecommons.org/licenses/by/4.0), which permits unrestricted use, distribution, and reproduction in any medium, provided the original work is properly cited.

The license is subject to the Beilstein Journal of Nanotechnology terms and conditions: (http://www.beilstein-journals.org/bjnano)

The definitive version of this article is the electronic one which can be found at: doi:10.3762/bjnano.8.81

\section{References}

1. Pan, D. Z.; Liebmann, L.; Yu, B.; Xu, X.; Lin, Y. Pushing multiple patterning in sub-10nm: are we ready?. In Proceedings of the 52nd Annual Design Automation Conference, San Francisco, CA, U.S.A., June 7-11, 2015; ACM: New York, NY, U.S.A.; No. 197. doi:10.1145/2744769.2747940 